\title{
Epireflection operators vs perfect morphisms and closed classes of epimorphisms
}

\section{G.E. Strecker}

\begin{abstract}
Operators are defined that yield basic Galois closure operations for almost every category. These give rise to a new and more general approach for characterization of epireflective subcategories, and construction of epireflective hulls. As a by-product, satisfactory characterizations of classes of perfect morphisms and w-extendable epimorphisms are obtained. Detailed proofs and examples will appear elsewhere.
\end{abstract}

\section{Introduction}

Characterizations of epireflective subcategories of categories with sufficiently nice completeness and smallness conditions, as well as knowledge of the existence of epireflective hulls and methods of constructing these hulls, have been known for some time ([2], [3], [4]). Full, isomorphism-closed, epireflective subcategories are usually characterized as those that are closed under the formation of products and some particular type of subobject (of a sufficiently strong type), and the epireflective hull of a class of objects is usually constructed by taking all "strong" subobjects of products of objects from the class. Classic examples are the corpact Hausdorfe spaces (which is the epireflective hul of the closed unit interval in the category of Hausdorff spaces) and the torsion free abelian groups (which is the epireflective hull of the integers in the category of abelian groups). The purpose of this paper is

Received 8 June 1972. 
to announce results that show that similar satisfactory characterizations and constructions can be obtained in a much more general setting; for example, in a category which does not even have products. We do so by introducing some general operators that exist for every category, regardless of its (co)completeness or smallness conditions, and show that these yield a fundamental Galois connection in the presence of the extremely weak (and, thus, usually satisfied) condition of the existence of either a terminal object or pushouts. Normally, under the connection, the "closed" classes of objects are precisely the object classes of full, isomorphismclosed epireflective subcategories, and the epireflective hull of a class of objects can be found by taking its "Galois closure".

\section{Definitions and notations (for any category C )}

By a subcategory of $C$ we shall always mean full, isomorphism-closed, subcategory. We shall say that $\mathrm{C}$ has weak sink pairs provided that for eacb pair of morphisms $(f, g)$ with common domain, there exists a pair of morphisms $(h, k)$ such that $h f=k g$. Note that this is an extremely weak condition. For example, it holds in any category which has either a terminal object or (weak) pushouts. The dual notion is called weak source pair.

We shall let Ob (respectively Mor, Iso, Epi, Mono) stand for the class of all objects (respectively morphisms, isomorphisms, epimorphisms, monomorphisms) of $C$. Also, for any class $\beta$ we shall let $P(\beta)$ denote all of the subclasses of $\beta$ (partially ordered by Inclusion). In particular, members of $P(B)$ may be proper classes.

We define $\Lambda: P($ Mor $)+P($ Mor $)$ by:

$\Lambda(\alpha)=\{f \in \operatorname{Mor} \mid$ for each $g \in \alpha$ and all morphisms $h$ and $k$ for which $h g=f k$, there exists some morphism $d$ such that $d g=k$ and $f d=h$ l.

Dually $T=\Lambda^{\text {op }} . \Lambda(\alpha)$ (respectively $T(\alpha)$ ) is called the class of $\alpha$-Zower (respectively $\alpha$-upper) diagonalizable morphisms.

We define $X: P(O b) \rightarrow P($ Mor $)$ by:

$X(\omega)=\{f: A \rightarrow B \mid$ for each $K \in \omega$ and each $g: A \rightarrow K$, there is some $h: B \rightarrow K$ such that $g=h f\}$. 
Dually $L=X^{\text {op }}$. $X(\omega)$ (respectively $L(\omega)$ ) is called the class of all $\omega$-extendable (respectively $\omega$-liftable) morphisms.

We define $E: P($ Mor $) \rightarrow P($ Epi $)$ and $M: P($ Mor $) \rightarrow P($ Mono $)$ by:

$$
E(\alpha)=\alpha \cap \text { Epi and } M(\alpha)=\alpha \cap \text { Mono . }
$$

We define $D: P($ Mor $) \rightarrow P(0 b)$ by:

$D(\alpha)=\{A \in O b \mid$ every morphism with domain $A$ is in $\alpha\}$.

Dually $C=D^{\circ \mathrm{P}} . \quad D(\alpha)$ (respectively $C(\alpha)$ ) is called the class of $\alpha$-domain (respectively $\alpha$-codomain) objects.

In [5] Kelley has defined strong epimorphisms to be the members of ET(Mono) and strong monomorphisms to be the members of $M \Lambda$ (Epi). Also, for any class of objects, $\omega$, Herrlich [2] has defined the w-perfect morphisms to be the members of $\Lambda E X(\omega)$. We shall use these terminologies and shall also call a class, $\alpha$, a perfect class of morphisms provided that for some $\omega, \alpha=\Lambda E X(\omega)$.

We shall call $\alpha \subset$ Mor a fundcomental class of morphisms provided that $\alpha:$

$F(1)$ : contains all strong monomorphisms,

$F(2)$ : is closed under composition,

$F(3)$ : is closed under the formation of pullbacks ${ }^{1}$,

$F(4)$ : is closed under the formation of multiple pullbacks,

$F(5)$ : is closed under the formation of products, and

$F(6)$ : is left-cancellative; that is, if $g f \in \alpha$, then $f \in \alpha$.

Dual notion: cofundomental class of morphisms.

We shall call $\alpha \subset$ Epi a standard class of epimorphisms provided that $\alpha:$

S(1): contains all isomorphisms,

S(2): is closed under composition,

1 When we say that a certain class of objects or morphisms is closed under the formation of a particular limit or colimit, we do not mean to imply that such limits or colimits necessarily exist; that is, there are no general completeness or cocompleteness conditions assumed. 
$S(3)$ : is closed under the formation of pushouts,

$S(4)$ : is closed under the formation of multiple pushouts, and

$S(5)$ : is left-cancellative with respect to epimorphisms; that is, if $g f \in \alpha$ and $f \in$ Epi, then $f \in \alpha$.

Dual notion: standard class of monomorphisms.

We shall call $\omega \subset O b$ a normal class of objects provided that $\omega$ :

$N(1)$ : is isomorphism-closed,

$N(2)$ : is closed under the formation of products, and

$N(3)$ is closed under the formation of strong subobjects.

Dual notion: conormal class of objects.

Notice that Mono is a fundamental class of morphisms, Epi is a standard class of epimorphisms, and Iso is fundamental and cofundamental as well as a standard class of epimorphisms and a standard class of monomorphisms. Also if $\mathrm{C}$ has pullbacks and coequalizers and if the class of regular monomorphisms is closed under composition, then it is fundamental. Also note that if a class of epimorphisms is cofundamental, then it is nearly standard. However, it may fail to satisfy $F(6)$; the class of all quotient maps in the category of topological spaces being a case in point. It is well-known that the class of objects of any epireflective subcategory is a normal class of objects. We will expand on this in Theorem 3 below.

\section{Results (for any category C )}

PROPOSITION 1. For any $a \subset$ Mor :

(i) $\Lambda(\alpha)$ satisfies $S(1), F(2), F(3), F(4)$, and $F(5)$,

(ii) if $\alpha \subset$ Epi, then $\Lambda(\alpha)$ is a fundomental class of morphisms,

(iii) $\Lambda(\alpha) \cap \alpha \subset$ Iso, and if Iso $\subset \alpha$, then $\Lambda(\alpha) \cap \alpha=$ Iso,

(iv) if $\alpha \subset$ Epi and $C$ has weak sink pairs, then $D \Lambda(\alpha)$ is a normal class of objects,

(v) $\alpha \subset T \Lambda(\alpha) \cap \Lambda T(\alpha)$, and

(vi) if $\beta \subset \alpha$, then $\Lambda(\alpha) \subset \Lambda(\beta)$ and $T(\alpha) \subset T(\beta)$. 
For any $\omega \subset \mathrm{Ob}$ :

(vii) $X(\omega)$ satisfies $s(1), s(2), s(3), s(4)$ and $F(6)$, and

(viii) EX(w) is a standard class of epimorphisms.

One can obtain as immediate corollaries of the above proposition many of the results concerning the properties of strong epimorphisms, $\omega$-perfect morphisms, and wextendable epimorphisms found in [5] and [2]. Notice that parts $(v)$ and $(v i)$ of the proposition imply that for any category, $\Lambda$ and $T$ provide a Galois connection between $P($ Mor) and itself. of more interest are other Galois connections that we will presently obtain.

$$
P(\text { Epi }) \underset{E X}{\stackrel{D \Lambda}{\rightleftarrows}} P(0 \mathrm{~b}) \underset{M L}{\stackrel{C T}{\rightleftarrows}} P(\text { Mono }) \text {. }
$$

LEMMA 1.

(i) $D \Lambda$ and $E X$ are inclusion reversing operators.

(ii) $\operatorname{D\Lambda EX}(\omega) \supset \omega$, for each $\omega \subset 0 \mathrm{~b}$.

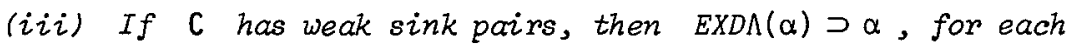
$\alpha \subset$ Epi .

THEOREM 1. For any category with weak sink pairs, EX and $D \Lambda$ yield a Galois connection (I) between $P(O b)$ and $P(E p i)$, and, dually, for any category with weak source pairs, $M L$ and $C T$ yield a Galois connection (II) between $P(O b)$ and $P($ Mono).

Following usual Galois terminology, we shall cell $\alpha \subset$ Epi a closed class of epimorphisms provided that $\alpha=\operatorname{EXD\Lambda }(\alpha)$ and $\omega \subset 0 \mathrm{~b}$ a closed class of objects provided that $\omega=D \Lambda E X(\omega)$. Likewise $\alpha \subset$ Mono wi $\perp$ be called a closed class of monomorphisms iff $\alpha=M L C T(\alpha)$ and $\omega \subset 0 \mathrm{~b}$ a coclosed class of objects iff $\omega=C T M L(\omega)$. Notice that, by Proposition $I$, closed classes of epimorphisms (monomorphisms) are standard, and, for most categories, (co)closed classes of objects are (co)normal. Thus Theorem 1 provides a very general setting for obtaining "(co)completions" of classes of objects - or of morphisms - (namely, by taking their (co)closures). It should be emphasized that these processes are valid even when the categories in question possess no completeness, cocompleteness or smallness conditions, except the rudimentary existence of weak sink (or 
source) pairs. For example, the above theory is "at home" in most of the categories in analysis (for example, Banach spaces), where arbitrary products need not exist, or in certain non-full subcategories of topological spaces, such as $H$-closed spaces and $p$-maps [1], which is neither complete nor cocomplete (but which has both an initial and a terminal object).

Herrlich [2], in the setting of a complete, cocomplete, well-powered and co-well-powered category, has obtained a characterization for the closed classes of epimorphisms; that is, the classes that are $E X(\omega)$ for some $\omega$. Below we obtain a somewhat simpler characterization under weaker hypotheses.

THEOREM 2. If $\mathrm{C}$ has multiple pushouts and is co-well-powered, then

(i) for each $\omega \subset 0 b, C$ is uniquely $(E X(\omega), \Lambda E X(\omega))-$ factorizable; that is, each morphism, $f$, has an essentially unique factorization $f=h g$, where $g$ is an w-extendable epimorphism and $h$ is an w-perfect morphism;

(ii) the closed classes of epimorphisms of $\mathrm{C}$ are precisety the standard classes of epimorphisms.

Thus, for reasonably nice categories, we have a myriad of various types of "canonical factorizations" and an internal characterization for the closed classes of epimorphisms. Below we show that under certain conditions there is a similar internal characterization for closed classes of objects.

THEOREM 3. Let $\omega$ be any class of objects of $\mathrm{C}$ and consider the following statements:

(a) $w$ is isomorphism-closed, and the full subcategory of $C$ with object class $\omega$ is epireflective in $C$;

(b) $\omega$ is a closed class of objects;

(c) $\omega$ is a normal class of objects.

(i) Always $(a) \Rightarrow(b)$ and $(a) \Rightarrow(c)$.

(ii) If $C$ has weak sink pairs, then $(b) \Rightarrow(c)$.

(iii) If $C$ has multiple pushouts and is co-well-powered, then 


$$
\begin{aligned}
& \text { (a) } \Leftrightarrow(b) \text {. } \\
& \text { (iv) If } C \text { is cocomplete, has products, and is co-well-powered, } \\
& \text { then }(a) \Leftrightarrow(b) \Leftrightarrow(c) \text {. }
\end{aligned}
$$

The equivalence of $(a)$ and (c) under hypotheses similar to those stated in (iv) above has been known for some time. However, part (iii) provides a new setting for the existence of epireflective hulls under quite weak hypotheses, namely:

COROLLARY 1. Every class, $\omega$, of objects of a co-well-powered category with multiple pushouts has con epireflective hull; that is, a smallest epireflective subcategory containing it. Moreover this hull is formed by taking the closure of $w$ according to the Galois connection (I) described above.

We now turn our attention to a somewhat different arrangement of the operators defined above.

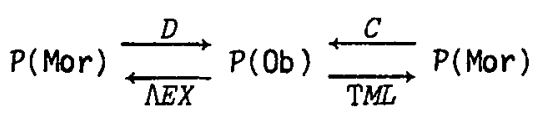

and note that $D$ and $\Lambda E X$ are always inclusion preserving and that if $C$ has weak sink pairs, then $\Lambda E X D$ is an idempotent operator. This, then, answers the open question in [2] of finding a characterization for perfect morphisms. Namely, for categories with weak sink pairs, $\alpha$ is a perfect class of morphisms iff $\alpha=\Lambda E X D(\alpha)$. Below we obtain an internal characterization under more restrictive hypotheses.

THEOREM 4. If $\mathrm{C}$ is complete, cocomplete, and co-well-powered, and $\alpha$ is a class of morphisms that satisfies $F(1), F(2), F(4)$ and either $F(3)$ or $\mathrm{F}(6)$, then $\Lambda E X D(\alpha) \subset \alpha$. If, furthermore, $\alpha$ satisfies:

$$
P(1): \quad \alpha \cap \operatorname{EXD}(\alpha) \subset \text { Iso }
$$

then $\alpha=\Lambda E X D(\alpha)$.

The first part of the above theorem shows that, with the stated hypotheses, $\Lambda E X D$ is an "Interior operator" for fundamental classes of morphisms. Thus each fundamental class of morphisms has a "perfect impletion". The second part shows that for sufficiently nice categories, the classes of perfect morphisms are precisely the fundamental classes of morphisms that satisfy $P(1)$. The condition $P(1)$ cannot be deleted from 
this characterization since Mono is a fundamental class of morpnisms that is not perfect in any category where there is some monomorphism that is not a strong monomorphism.

\section{References}

[1] Douglas Harris, "Katětov extension as a functor", Math. Ann. 193 (1971), 171-175.

[2] Horst Herrlich, "A generalization of perfect maps", General topology and $i$ ts relations to modern analysis and algebra IV (Proceedings of the Third Prague Topological Symposium, 1971, to appear).

[3] Horst Herrlich and George E. Strecker, "Coreflective subcategories", Trons. Amer. Math. Soc. 157 (1971), 205-226.

[4] John R. Isbell, "Structure of categories", BuzZ. Amer. Math. Soc. 72 (1966), 619-655.

[5] G.M. Kelly, "Monomorphisms, epimorphisms, and pull-backs", $J$. Austral. Math. Soc. 9 (1969), 124-142.

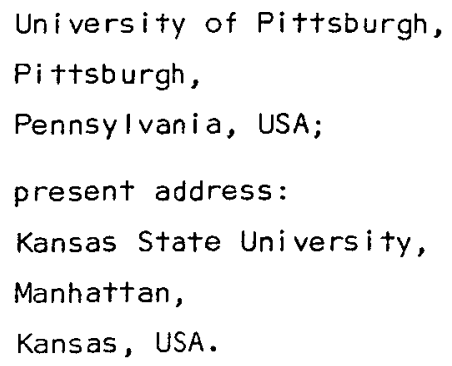

